\title{
Viral diversity limits immune diversity in asymptomatic phase of HIV infection
}

\author{
Shingo Iwami ${ }^{a}{ }^{*}$, Shinji Nakaoka ${ }^{b}{ }^{\dagger}$, Yasuhiro Takeuchi ${ }^{a}$ \\ Graduate School of Science and Technology, Shizuoka University, Japan ${ }^{a}$, \\ Aihara Complexity Modelling Project, ERATO, JST, The Tokyou University, Japan ${ }^{b}$
}

\begin{abstract}
We propose a new diversity threshold theory which states that the specific CTLs to the viral strain become inactivated (that is, some HIV strain can escape from its specific immune response) when the diversity of HIV strains exceeds some threshold number. We call this number "immune diversity threshold". Our theory can explain the inactivation of specific immune response and a limit of maximum immune diversity. We can conclude that the accumulation of viral diversity eventually leads to AIDS.
\end{abstract}

Keywords: HIV; Viral diversity; Immune diversity threshold; Frequency dependence

\section{Introduction}

The hallmark in HIV infection is a gradual decline of $\mathrm{CD} 4^{+} \mathrm{T}$ cells in the peripheral blood from 1000 cells per $\mu \mathrm{l}$ of blood to about 200 cells per $\mu \mathrm{l}$ at the onset of AIDS over a period that varies from several months to more than 10 years. CD4 ${ }^{+}$ T cells can be infected by HIV and may die, owing to a combination of the cytopathicity of the virus and immune elimination by cytotoxic T-lymphocytes (CTL) [6]. Antigen specific CD4 ${ }^{+} \mathrm{T}$ cells play a pivotal role in HIV infection. They provide helper activity to specific CTLs and to specific B cells, which may be responsible for control of HIV infection. Therefore, the loss of $\mathrm{CD}^{+} \mathrm{T}$ cells results in severe opportunistic infections. In particular the pathogens that are normally controlled in healthy individuals by $\mathrm{CD} 4^{+}$responses are responsible for morbidity and mortality

*This author is supported by Research Fellowships of the Japan Society for the Promotion of Science for Young Scientists.

${ }^{\dagger}$ This work was partly supported by the Sasakawa Scientific Research Grant from The Japan Science Society. 
in AIDS [14]. The HIV-1 genome is organized into three structural major genes (gag, pol, env), four accessory genes (vif, vpr, vpu, nef), and two regulatory genes (tat and rev). Gag codes for internal code protein; pol codes for the enzymatic protein reverse transcriptase, integrase and protease; env encodes the surface envelope glycoproteins gp120/gp41 [5]. HIV-1 cell tropisms are partly determined by the hypervariable loops V1/V2 and V3 in gp120, which also contain epitopes for neutralization antibodies. Furthermore, V3-loop peptides have been shown to be presented by the major histocompatibility complex (MHC) class I and class II in humans and are recognized by CTLs and by T helper cells. Thus, amino acid changes in V3 that confer escape from neutralization or tropism changes might also result in escape from cellular immunity such as CTL [15]. There is increasing evidence that CTLs play a central role in the immune response to HIV. They are activated in vivo to high levels at the same time as the initial clearance of the primary viremia. It is also clear that they are important in the maintenance of the asymptomatic phase of infection before the development of AIDS [7].

It is said that patients infected with HIV-1 have a long and variable incubation period between infection and the development of AIDS. This incubation period is characterized by slowly decreasing number of $\mathrm{CD} 4^{+} \mathrm{T}$ cells. Many studies have shown that viral diversity increases in the asymptomatic phase by the mutational escape of HIV-1. An error-prone nature of HIV reverse transcriptase contributes to the tremendous diversity of HIV [15]. Under the pressure of CTL responses, viruses that contain mutated critical amino acids in epitopes recognized by CTL are selected [17], [26]. The possible importance of viral diversity for the disease progression and the development of AIDS has been very well worked out in the early 1990s, especially by some important works of Martin A, Nowak. In [18]-[23], Nowak et al. constructed simple mathematical models which can explain that the disease progression and development of AIDS are caused by viral diversity. These interesting and elegant studies revealed the importance of viral diversity, in particular, the potential existence of viral diversity threshold which states that the viral load explodes when the diversity of virus strains exceeds this threshold number. However, in the theory, a constant (background) replication of antigen and immune impairment are essential factor for the explosion (Note that the diversity theory may not hold if the replication is dependent on $\mathrm{CD} 4^{+} \mathrm{T}$ cells). In fact, viral replication is restricted by $\mathrm{CD} 4^{+}$ $\mathrm{T}$ cells dynamics in HIV infection. Therefore, we should consider the dynamics with the immune effect of $\mathrm{CD} 4^{+} \mathrm{T}$ cell.

In this paper, we propose a new diversity threshold theory which states that the specific CTLs to some viral strain become inactivated (that is, some HIV strain can 
escape from its specific immune response) when the diversity of HIV strains exceeds some threshold number. We call this number "immune diversity threshold". In terms of our mathematical model the threshold is given by a capacity of immune response. During asymptomatic phase viral diversity is accumulated by the mutational escape of HIV and this accumulation leads to an escape of the nonmutational existing strain from its specific immune response. Although several hypotheses such as sequestration, HLA down-regulation, exhaustion of CTL and FasL expression have been proposed to explain the escape of HIV-1 from CTL in [16], our theory proposes a new hypothesis which explains the escape of the strain caused by viral diversity. Further, some immune response specific to the HIV strain becomes inactivated in the end of asymptomatic phase of HIV infection. This may imply that the escape of the strain denotes an exhaustion of immune cells. We will discuss whether viral diversity is a cause of AIDS and why AIDS eventually develops.

\section{Mathematical model}

The viral diversity increases and is accumulated during HIV infection (in particular, during asymptomatic phase). Therefore we must adopt the diversity effect when we consider HIV dynamics in asymptomatic phase. In [8], we proposed the following mathematical models in which CTL reactions (CTL proliferation and elimination of infected cells by CTLs) depend on the frequency of productively infected cells:

$$
\begin{aligned}
& T^{\prime}=\lambda-d T-\sum_{l=1}^{n} \beta_{l}^{\prime} T V_{l}, \\
& I_{j}^{\prime}=\beta_{j}^{\prime} T V_{j}-a I_{j}-q Z_{j} \frac{I_{j}}{T+\sum_{l=1}^{n} I_{l}}, \\
& V_{j}^{\prime}=k a I_{j}-u V_{j}, \\
& Z_{j}^{\prime}=c Z_{j} \frac{I_{j}}{T+\sum_{l=1}^{n} I_{l}}-\delta Z_{j} . \quad(j=1,2, \ldots, n)
\end{aligned}
$$

This model consists of $3 n+1$-variables: $T$ denotes the population size of uninfected $\mathrm{CD} 4^{+} \mathrm{T}$ cells, $I_{j}$ denotes one of $\mathrm{CD} 4^{+} \mathrm{T}$ cells infected with virus particle of type $j, V_{j}$ denotes one of the free virus particle of type $j$, and $Z_{j}$ denotes one of CTLs of type $j$, respectively. The parameter $\lambda$ is a rate at which new target cells are generated. Uninfected cells, infected cells, virus and CTLs are assumed to die at respective rates $d, a, u$ and $\delta$. In particular, $a$ represents the sum of natural death of infected $\mathrm{CD}^{+} \mathrm{T}$ cell and additional death due to viral growth inside infected CD4 ${ }^{+}$ $\mathrm{T}$ cell. Once cells are infected, we assume that they produce $k$ new virus particles during their life, which on average has a length $1 / a$. 
In addition, we notice the asymmetric interaction between HIV strains and the immune cells: different virus strains can infect $\mathrm{CD} 4^{+} \mathrm{T}$ cells irrespective of the strain's specificity, but specific CTLs are only activated by its specific HIV strain. Naturally this asymmetric interactions become remarkable as the diversity increases. Therefore, we chose one of the simplest form which emphasizes the asymmetric interactions between HIV strains and immune responses. This effect is reflected in a rate of proliferation of CTLs and a rate of elimination of infected cells by CTLs. For simplification, we assume that the number of unit CTL encountering all CD4 ${ }^{+} \mathrm{T}$ cells $\left(T, I_{1}, \ldots, I_{n}\right)$ is always constant. For example, CTL proliferation in our model is given by $\frac{c Z_{j} I_{j}}{T+\sum_{l=1}^{n} I_{l}}$. The parameter $c$ is a product of the CTL responsiveness and the number of unit CTL encountering all $\mathrm{CD}^{+} \mathrm{T}$ cells. Remark that (1) has the frequency dependent term, $\frac{I_{j}}{T+\sum_{l=1}^{n} I_{l}}$, characterized by viral diversity. Here the frequency describes the probability that an HIV specific CTL encounters the corresponding specific infected cells. Actually CTL interacts with many infected cells, decides whether its target is specific or not during a couple of minutes and kills the infected cells if they are its specific cells. Thus CTLs recognize their specific cells through the random interactions [3], [4]. Therefore the CTL proliferation can be considered as some function of the frequency of encounters. In the similar manner, the specific infected cells are killed by specific CTLs at a rate $\frac{q Z_{j} I_{j}}{T+\sum_{l=1}^{n} I_{l}}$ via the interactions between CTLs and infected cells. The parameter $q$ is a product of a rate at which CTLs kill infected cells and the number of unit CTL encountering all $\mathrm{CD}^{+} \mathrm{T}$ cells. We remark that we can use the same term for describing different CTL reactions because CTL proliferation and lytic effect mechanism are based on the random interactions. Further in our model, high uninfected CD4 ${ }^{+} \mathrm{T}$ cells count results in low CTL proliferation and lytic effect. Since CTL specific interactions decrease by the frequency dependence terms if uninfected $\mathrm{CD} 4^{+} \mathrm{T}$ cells count is high, the antigen stimulation to CTL decreases and this shortage leads to the negative effect for the CTL reactions. This is because we neglect the helper effects from uninfected CD4 ${ }^{+} \mathrm{T}$ cells to CTLs as well as many HIV models do [23]. Although the negative effect becomes weak if we include the helper effect in the CTL proliferation term, we leave the inclusion of this additional effect as a future work and investigate the effect of the frequency terms in this paper. On the other hand, the rate of infection is given by $\beta_{j}^{\prime} T V_{j}$ which is proportional to the amount of HIV strain $j$ where the parameter $\beta_{j}^{\prime}$ is a product of the infection rate of HIV strain $j$ and the number of unit HIV strain $j$ encountering unit uninfected CD4 ${ }^{+} \mathrm{T}$ cells. This form implies that the number of unit HIV encountering all uninfected $\mathrm{CD} 4^{+} \mathrm{T}$ cells is a linear function of $T$. Thus our mathematical model can characterize the asymmetric 
interactions emphasized by viral diversity.

Here we do not use different parameters for bursting ( $a$ and $k$ ), lytic effect $(q)$, CTL proliferation $(c)$, and death rate $(u$ and $\delta)$ in order to obtain the clear results and simplify the model. However, these assumptions are not essential for our findings. Even if we use the different parameters, we can obtain similar implications for HIV infection (see Discussion).

Further, we can reduce model (1) to a simpler form, using a quasi-steady-state approach and some scaling parameters, as follows (See [8]);

$$
\begin{aligned}
& T^{\prime}=1-T-\sum_{l=1}^{n} \beta_{l} T I_{l}, \\
& I_{j}^{\prime}=\beta_{j} T I_{j}-a I_{j}-q Z_{j} \frac{I_{j}}{T+\sum_{l=1}^{n} I_{l}}, \\
& Z_{j}^{\prime}=c Z_{j} \frac{I_{j}}{T+\sum_{l=1}^{n} I_{l}}-\delta Z_{j}, \quad(j=1,2, \ldots, n) .
\end{aligned}
$$

In (2) we use scaling notations. Note that, as same as (1), the interactions between specific infected cells and specific CTLs depend on the frequency that specific CTLs encounter the specific infected cells, but the interactions between uninfected cells and specific infected cells are not frequency dependent.

\section{Threshold theory in asymptomatic phase}

We introduce a basic reproductive number $R_{j}$, which is defined as the number of newly infected cells that arise from any one infected cell when all cells are uninfected. A rate at which one infected cell gives rise to new infected cells is given by $\beta_{j} T$. If all cells are uninfected, then $T=1$. Since the average life-time of an infected cell is $1 / a$, we obtain

$$
R_{j}=\frac{\beta_{j}}{a} .
$$

For our convenience, let $\beta_{1}<\beta_{2}<\ldots<\beta_{n}$ without loss of generality. Then the basic reproductive numbers satisfy the following relation:

$$
R_{1}<R_{2}<\ldots<R_{n}
$$

Further we assume $R_{1}>1$, which leads to the persistence of HIV strain 1 .

System (2) has various equilibria and the maximum number of nonnegative equilibria of (2) is given by the following expression:

$$
\left({ }_{n} C_{n}+{ }_{n} C_{n-1}+\ldots+{ }_{n} C_{1}\right)+\left({ }_{n} C_{n} \cdot{ }_{n} C_{1}+{ }_{n} C_{n-1} \cdot{ }_{n-1} C_{1}+\ldots+{ }_{n} C_{1} \cdot{ }_{1} C_{1}\right)+1
$$

and these equilibria can be divided into three types (i)(ii)(iii): 
(i) Controlled equilibrium: $E_{c}$-type, which means that all specific immune strains are activated by their corresponding HIV strains. For example, $E_{c}$-type equilibrium can include the following equilibrium:

$$
\left(\tilde{T} ; \tilde{I}_{1}, \ldots, \tilde{I}_{k}, 0, \ldots, 0 ; \tilde{Z}_{1}, \ldots, \tilde{Z}_{k}, 0, \ldots, 0\right) .
$$

(ii) Uncontrolled equilibrium : $E_{u}$-type, which means that one of all specific immune strains becomes inactivated in spite of the existence of its specific HIV strain but the other immune strains are activated by their corresponding HIV strains. For example, $E_{u}$-type equilibrium can include the following equilibrium:

$$
\left(\hat{T} ; \hat{I}_{1}, \ldots, \hat{I}_{k}, 0, \ldots, 0 ; 0, \hat{Z}_{2}, \ldots, \hat{Z}_{k}, 0, \ldots, 0\right) .
$$

(iii) Healthy equilibrium : $E_{h}$-type, which means that all HIV strains do not exist.

From the definitions of $E_{c}$-type and $E_{u}$-type, the numbers of $E_{c}$-type and $E_{u}$-type equilibria in $\mathbb{R}_{+}^{2 n+1}$ are ${ }_{n} C_{n}+{ }_{n} C_{n-1}+\ldots+{ }_{n} C_{1}$ and ${ }_{n} C_{n} \cdot{ }_{n} C_{1}+{ }_{n} C_{n-1} \cdot{ }_{n-1} C_{1}+\ldots+$ ${ }_{n} C_{1} \cdot{ }_{1} C_{1}$, respectively. Further, the number of equilibria which can exist in $\mathbb{R}_{+}^{2 n+1}$ is given by the following expression:

$$
\left({ }_{n} C_{n}+{ }_{n} C_{n-1}+\ldots+{ }_{n} C_{1}\right)+\left(\frac{{ }_{n} C_{n} \cdot{ }_{n} C_{1}}{1}+\frac{{ }_{n} C_{n-1} \cdot{ }_{n-1} C_{1}}{2}+\ldots+\frac{{ }_{n} C_{1} \cdot{ }_{1} C_{1}}{n}\right)+1,
$$

because $R_{1}<R_{2}<\ldots<R_{n}$. Here we remark that there does not exist uncontrolled equilibrium in which more than two immune strains are inactivated while their corresponding viral strains exist because of competitive exclusion for the viral strains.

For system (2), we consider the following equilibrium:

$$
E_{n}^{+}=\left(T^{+} ; I_{1}^{+}, \ldots, I_{n}^{+} ; Z_{1}^{+}, \ldots, Z_{n}^{+}\right),
$$

where

$$
\begin{aligned}
T^{+} & =\frac{2}{1+\sqrt{1+\frac{4 \delta \sum_{l=1}^{n} \beta_{l}}{c-n \delta}}}, \\
I_{1}^{+} & =\ldots=I_{n}^{+}=\frac{-1+\sqrt{1+\frac{4 \delta \sum_{l=1}^{n} \beta_{l}}{c-n \delta}}}{2 \sum_{l=1}^{n} \beta_{l}} \\
Z_{j}^{+} & =\frac{\left(\beta_{j} T^{+}-a\right)\left(T^{+}+\sum_{l=1}^{n} I_{l}^{+}\right)}{q},
\end{aligned}
$$

for $j=1, \ldots, n$. It is clear that this equilibrium belongs to $E_{c}$-type. We remark that the infected cell strains have the different concentration if immune ability such 
as $c$ and $\delta$ for each immune cell are not the same. For simplification we use the same parameters for immune ability in this analysis because the difference does not change our main results.

Another equilibrium is

$$
\bar{E}_{n}=\left(\bar{T} ; \bar{I}_{1}, \ldots, \bar{I}_{n} ; 0, \bar{Z}_{2}, \ldots, \bar{Z}_{n}\right)
$$

where

$$
\begin{aligned}
& \bar{T}=\frac{a}{\beta_{1}}, \\
& \bar{I}_{2}=\ldots=\bar{I}_{n}=\frac{1-\bar{T}+\beta_{1} \bar{T}^{2}}{\left\{\sum_{l=2}^{n} \beta_{l}+\beta_{1}\left(\frac{c}{\delta}-(n-1)\right)\right\} \bar{T}}, \\
& \bar{I}_{1}=\left(\frac{c}{\delta}-(n-1)\right) \bar{I}_{j}-\bar{T} \\
& \bar{Z}_{j}=\frac{c\left(\beta_{j} \bar{T}-a\right)}{q \delta} \bar{I}_{j}
\end{aligned}
$$

for $j=2, \ldots, n$ and $n \geq 2$. It is clear that this equilibrium belongs to $E_{u}$-type.

The existence condition of these equilibria is as follows:

$$
\begin{aligned}
& E_{n}^{+} \in \mathbb{R}_{+}^{2 n+1} \Longleftrightarrow \frac{1+\sqrt{1+\frac{4 \delta\left(\beta_{1}+\beta_{2}+\ldots+\beta_{n}\right)}{c-n \delta}}}{2}<R_{1}, \\
& \bar{E}_{n} \in \mathbb{R}_{+}^{2 n+1} \Longleftrightarrow \frac{1+\sqrt{1+\frac{4 \delta\left(\beta_{2}+\beta_{3}+\ldots+\beta_{n}\right)}{c-(n-1) \delta}}}{2}<R_{1} .
\end{aligned}
$$

In the above two inequalities, the left hand side in the former inequality is larger than one in the latter when $c-n \delta>0$. This implies that $\bar{E}_{n}$ exists if so does $E_{n}^{+}$.

It is easy to check that for the Jacobian matrix evaluated at $\bar{E}_{n}$, the transversal eigenvalue of $Z_{1}$-direction is given by $c \bar{I}_{1} /\left(\bar{T}+\sum_{l=1}^{n} \bar{I}_{l}\right)-\delta$. If $c \bar{I}_{1} /\left(\bar{T}+\sum_{l=1}^{n} \bar{I}_{l}\right)-\delta>$ 0, then $\bar{E}_{n}$ is unstable. Remark that this equilibrium can be stable with reasonable conditions. By substituting $\bar{T}$ and $\bar{I}_{1}, \ldots, \bar{I}_{n}$ into the above inequality, we obtain that

$$
\begin{aligned}
\frac{c \bar{I}_{1}}{\bar{T}+\sum_{l=1}^{n} \bar{I}_{l}}-\delta>0 & \Longleftrightarrow \bar{I}_{1}>\bar{I}_{2}=\ldots=\bar{I}_{n} \\
& \Longleftrightarrow R_{1}>\frac{1+\sqrt{1+\frac{4 \delta\left(\beta_{1}+\beta_{2}+\ldots+\beta_{n}\right)}{c-n \delta}}}{2} .
\end{aligned}
$$

Thus, if $R_{1}>\left(1+\sqrt{1+4 \delta\left(\beta_{1}+\beta_{2}+\ldots+\beta_{n}\right) /(c-n \delta)}\right) / 2$, then $\bar{E}_{n}$ is unstable. Remark that this condition corresponds to the existence condition of $E_{n}^{+}$. 
In the similar manner, all boundary equilibria are unstable whenever the interior equilibrium exists in $\mathbb{R}_{+}^{2 n+1}$. Therefore, our conjecture is that (2) is permanent whenever the interior equilibrium exists. To prove this is our future work. Further we can prove that two equilibria can not be stable simultaneously. For instance, we have obtained some detailed analysis of the existence and instability conditions of equilibria for $n=3$ in [9].

\subsection{The accumulation of viral diversity}

Consider population sizes of uninfected and infected $\mathrm{CD} 4^{+} \mathrm{T}$ cells at $E_{n}^{+}$. Assume the existence of interior equilibrium $E_{n}^{+}$. Then the total infected CD4 ${ }^{+} \mathrm{T}$ cell size can be given by the following equality:

$$
I(n)=\sum_{j=1}^{n} I_{j}^{+}=\frac{n\left(-1+\sqrt{1+\frac{4 \delta \sum_{l=1}^{n} \beta_{l}}{c-n \delta}}\right)}{2 \sum_{l=1}^{n} \beta_{l}} .
$$

Since $I(n) \rightarrow \infty$ as $n \rightarrow c / \delta, I(n+m)$ must be larger than $I(n)$ for sufficiently large integer $m$. This implies that the total infected $\mathrm{CD} 4^{+} \mathrm{T}$ cell size eventually increases with the viral diversity $n$. Remark that the total force of infection rate $\sum_{j=1}^{n} \beta_{j} I_{j}^{+}$ always increases with the viral diversity $n$, which is the same as the results obtained in [10], [11], [12]. From the expression of uninfected $\mathrm{CD}^{+} \mathrm{T}$ cell, let

$$
T(n)=T^{+}=\frac{2}{1+\sqrt{1+\frac{4 \delta \sum_{l=1}^{n} \beta_{l}}{c-n \delta}}} .
$$

It is clear that $T(n)$ is a decreasing function of an integer $n$. This implies that the uninfected $\mathrm{CD}^{+} \mathrm{T}$ cell size decreases with the viral diversity $n$. Consequently, the accumulation of viral diversity deteriorates symptoms of HIV infection if a solution converges to the equilibrium.

\subsection{The immune diversity threshold}

In [18]-[23], Nowak et al. proposed "antigen diversity threshold theory" which states that the viral load explodes when the diversity of HIV strains exceeds the threshold number. This theory is very elegant and captures an essential evolutionary mechanism underlying HIV disease progression. In this study, we propose "immune diversity threshold theory" which states that the specific CTLs to some viral strain become inactivated (that is, some HIV strain can escape from its specific immune 
response) when the diversity of HIV strains exceeds the threshold number. Our theory can explain the inactivation of specific immune response and a limit of immune diversity.

The equilibrium of (2) must satisfy

$$
\begin{aligned}
& 1-T-\sum_{l=1}^{n} \beta_{l} T I_{l}=0, \\
& \beta_{j} T I_{j}-a I_{j}-q Z_{j} \frac{I_{j}}{T+\sum_{l=1}^{n} I_{l}}=0, \\
& c Z_{j} \frac{I_{j}}{T+\sum_{l=1}^{n} I_{l}}-\delta Z_{j}=0,
\end{aligned}
$$

for $j=1,2, \ldots, n$. If (2) has the controlled equilibrium $E_{n}^{+}$, then $Z_{j}^{+} \neq 0$ and the following equation should be satisfied from the third equation of $(5)$ for $j=1,2, \ldots, n$ :

$$
\frac{I_{j}^{+}}{T^{+}+\sum_{l=1}^{n} I_{l}^{+}}=\frac{\delta}{c} .
$$

Adding the above equations from $j=1$ to $n$ yields

$$
\sum_{l=1}^{n} I_{l}^{+}=\frac{n \delta}{c}\left(T^{+}+\sum_{l=1}^{n} I_{l}^{+}\right) .
$$

This is equivalent to

$$
\left(1-\frac{n \delta}{c}\right) \sum_{l=1}^{n} I_{l}^{+}=\frac{n \delta}{c} T^{+} .
$$

Since $T^{+}>0$ and $I_{j}^{+}>0(j=1,2, \ldots, n)$, the following threshold condition must be satisfied:

$$
1-\frac{n \delta}{c}>0
$$

However, for arbitrary fixed parameters $c$ and $\delta$, there exists some critical number

$$
n^{*}=\frac{c}{\delta}
$$

We call $n^{*}$ "immune diversity threshold". If $n \geq n^{*}$, then (6) can not be satisfied and the controlled equilibrium $E_{n}^{+}$vanishes from a nonnegative cone. What does this mean? What happens with $n>n^{*}$ ? We will consider the situation with $k<n^{*}<k+1$. Assume that

$$
R_{1}>\frac{1+\sqrt{1+\frac{4 \delta\left(\beta_{1}+\beta_{2}+\ldots+\beta_{k}\right)}{c-k \delta}}}{2} .
$$


If $n=k$, then $E_{k}^{+}$exists in $\mathbb{R}_{+}^{2 k+1}$ and $\bar{E}_{k}$ is unstable because $k<n^{*}$. After some time, the mutant HIV strain is introduced (i.e., $n=k+1$ ). Then $E_{k+1}^{+}$can no longer exist in $\mathbb{R}_{+}^{2(k+1)+1}$ because $n^{*}<k+1$. Furthermore, we assume that

$$
R_{1}>\frac{1+\sqrt{1+\frac{4 \delta\left(\beta_{2}+\ldots+\beta_{k}+\beta_{k+1}\right)}{c-k \delta}}}{2} .
$$

Then $\bar{E}_{k+1}$ exists in $\mathbb{R}_{+}^{2(k+1)+1}$. The transversal eigenvalue of $Z_{1}$-direction at $\bar{E}_{k+1}$ is

$$
\frac{\partial Z_{1}^{\prime}}{\partial Z_{1}}=\frac{c I_{1}}{T+\sum_{l=1}^{k+1} I_{l}}-\delta
$$

If $\bar{E}_{k+1}$ is stable, then the transversal eigenvalue is clearly negative at $\bar{E}_{k+1}$. Further, we can show that the transversal eigenvalue is also negative even if $\bar{E}_{k+1}$ is unstable. In fact, from a straightforward calculation at $\bar{E}_{k+1}$, we can obtain the following relation:

$$
\frac{c \bar{I}_{1}}{\bar{T}+\sum_{l=1}^{k+1} \bar{I}_{l}}-\delta=\delta\left(\frac{\bar{I}_{1}}{\bar{I}_{2}}-1\right) .
$$

Since $\bar{I}_{1}=(c / \delta-k) \bar{I}_{2}-\bar{T}$, we can obtain

$$
\frac{\bar{I}_{1}}{\bar{I}_{2}}=\left(\frac{c}{\delta}-k\right)-\frac{\bar{T}}{\bar{I}_{2}} .
$$

Therefore, the transversal eigenvalue is always negative even if $\bar{E}_{k+1}$ is unstable because $n^{*}<k+1$ :

$$
\frac{\partial Z_{1}^{\prime}}{\partial Z_{1}}=\delta\left(\frac{\bar{I}_{1}}{\bar{I}_{2}}-1\right)=\delta\left(\frac{c}{\delta}-(k+1)-\frac{\bar{T}}{\bar{I}_{2}}\right)<0,
$$

since $n^{*}=c / \delta<k+1$. This implies that if the number of HIV strains exceeds the immune diversity threshold, then $Z_{1}$ becomes always inactivated (i.e., the HIV strain 1 escapes from its specific immune response). We remark that $E_{k+l}^{+}(l \geq 1)$ and $\bar{E}_{k+m}(m \geq 2)$ can no longer exsist in a nonnegative cone if $k<n^{*}<k+$ 1. When a further additional mutant HIV strain is introduced (i.e., $n \geq k+2$ ), some $E_{u}$-type equilibrium bifurcates in a nonnegative cone (see Discussion). In the similar manner, we can show that the transversal eigenvalue of the inactivated HIV strain-direction is also negative at the $E_{u}$-type equilibrium. Therefore, we can conclude that the number of maximum immune diversity is $\left[n^{*}\right]$, where $\left[n^{*}\right]$ denotes the greatest integer less than $n^{*}$. This implies that the accumulation of viral diversity limits the immune diversity. Note that the maintenance of multiple HIV strains is due to the immune responses. If all immune cells are inactivated, then only one HIV strain with the maximum basic reproductive number can exist via competitive 
exclusion. Thus immune response regulates the exploitative competition among HIV strains and leads to viral diversity. This implies that immune response has two sides of the same coin; one is the control of viral replications and another is the accumulation of viral diversity. However, interestingly, the accumulation of viral diversity maintained by immune response eventually limits immune diversity. Note that this threshold number is only characterized by the parameters $c$ and $\delta$ expressing immune properties. In our mathematical model, the immune system limits its ability by themselves. In fact $1 / \delta$ denotes the average life-time of an immune cell and $c$ can be interpreted as the maximum efficiency of immune inducement, which imply that $n^{*}$ gives the maximum number of new immune cells that arise from any one immune cell. That is, $n>n^{*}$ implies that viral diversity exceeds immune ability. Actually, human immune system seems to have a limit and to give in viral diversity in HIV infection. In our theory, the HIV strain that has the minimum basic reproductive number can escape and its specific immune response becomes inactivated at first instead of invading the strain with the larger basic reproductive number. This is because a shortage of infected cell sneaks past the corresponding specific CTL response and underwhelms the immune response [28] (Note that $\bar{I}_{1}<\bar{I}_{j}, j=2, \ldots, n$ ). Our theory is based on some balance between the regulation of the exploitative competition among HIV strains and the shortage of antigen stimulation of specific immune response.

\subsection{Virtual experiment}

In this part, we simulate, using Mathematica, that the HIV strain with the minimum basic reproductive number can escape from its specific immune response if the number of HIV strains exceeds the immune diversity threshold.

We assume that HIV strain $I_{j}$ and its specific immune cell $Z_{j}(j=1, \ldots, 9)$ are present at first. We carry out a numerical simulation for (2) with parameters

$$
\left\{\begin{array}{l}
\beta_{1}=22.5, \beta_{2}=22.75, \beta_{3}=23, \beta_{4}=23.25, \beta_{5}=23.5, \beta_{6}=23.75, \beta_{7}=24, \\
\beta_{8}=24.25, \beta_{9}=24.5, a=1.2, q=15, c=10.8, \delta=1.1
\end{array}\right.
$$

In this case, the immune diversity threshold is given by

$$
n^{*}=\frac{c}{\delta} \approx 9.82
$$

Hence the current number of HIV strains $n=9$ does not exceed this threshold value (i.e., (6) is satisfied). Since

$$
\frac{1+\sqrt{1+\frac{4 \delta\left(\beta_{1}+\beta_{2}+\ldots+\beta_{9}\right)}{c-9 \delta}}}{2}=16.59<18.75=R_{1}=\beta_{1} / a,
$$



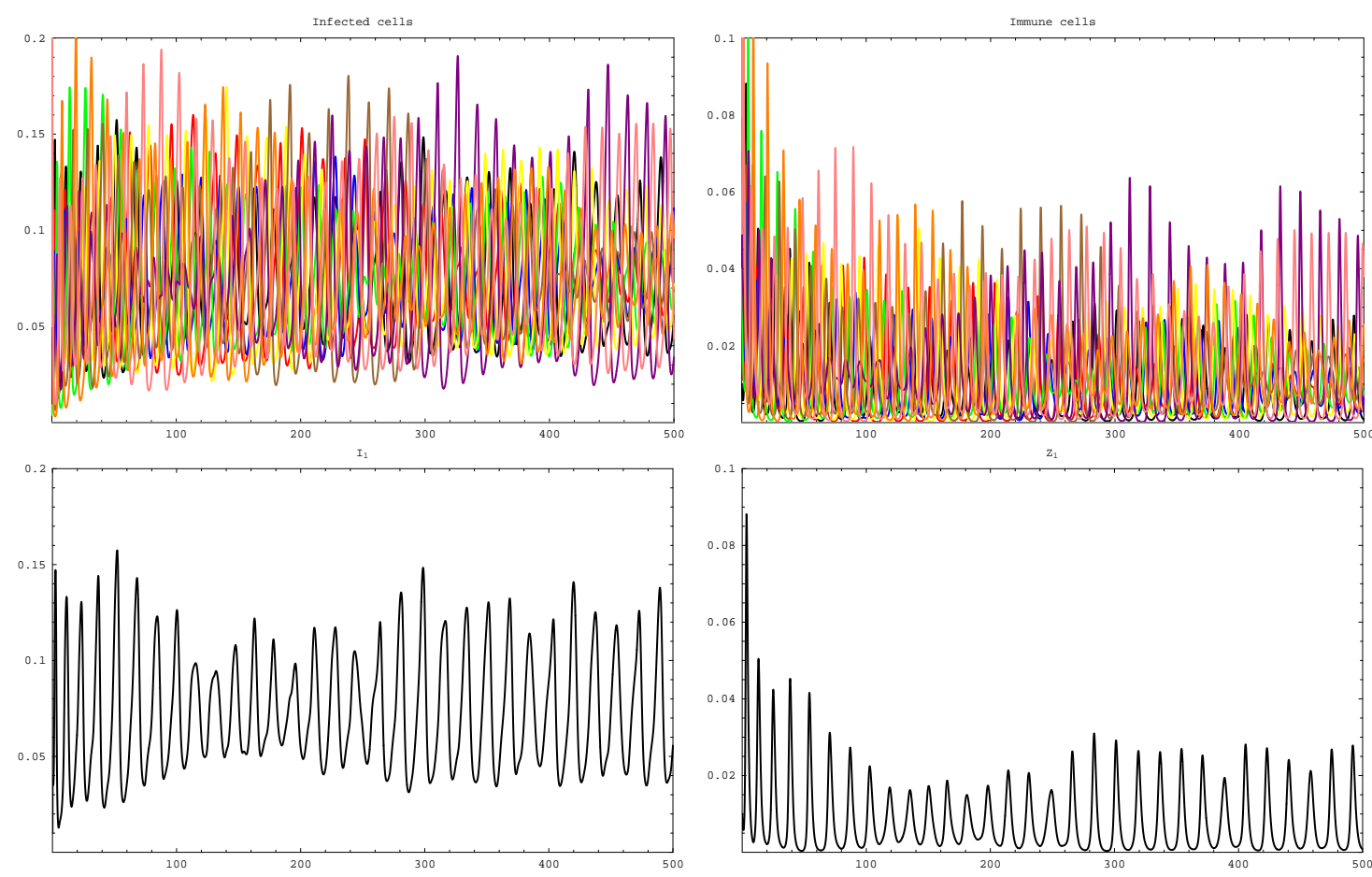

Figure 1: All HIV strains coexist and all specific immune responses are activated: HIV strain $I_{j}$ and its specific immune cell $Z_{j}(j=1, \ldots, 9)$ are present at first. In this case, $E_{9}^{+}$exists because $n<n^{*}$ is satisfied. The solution of (2) eventually converges to $E_{9}^{+}$. This implies that the immune system maintains immune diversity. Lower panels represent $I_{1}$ (left) and $Z_{1}$ (right). The horizontal axis represents time.

$E_{9}^{+}$exists in a nonnegative cone and the other equilibria are unstable. A computer simulation is shown in Fig.1. Although the dynamics looks like chaotic, the solution eventually converges to $E_{9}^{+}$. All HIV strains coexist and all specific immune responses are activated. That is, the immune system maintains immune diversity.

After some time, we assume that a mutant HIV strain $I_{10}$ is introduced by an evolutionary event of disease progression. An infection rate of the mutant is set as $\beta_{10}=24.75$ (although we assume that the infection rate is the maximum, it is not the essential assumption for our result). This evolutionary event breaks the condition (6) because the number of HIV strains $n=10$ exceeds the immune diversity threshold, $n^{*}$. Then controlled equilibrium $E_{10}^{+}$can not exist in the nonnegative cone. However, the existence condition of $\bar{E}_{10}$ is satisfied because

$$
\frac{1+\sqrt{1+\frac{4 \delta\left(\beta_{2}+\ldots+\beta_{9}+\beta_{10}\right)}{c-9 \delta}}}{2}=16.67<18.75=R_{1} .
$$

This implies that $\bar{E}_{10}$ exists in the nonnegative cone and the other equilibria are 

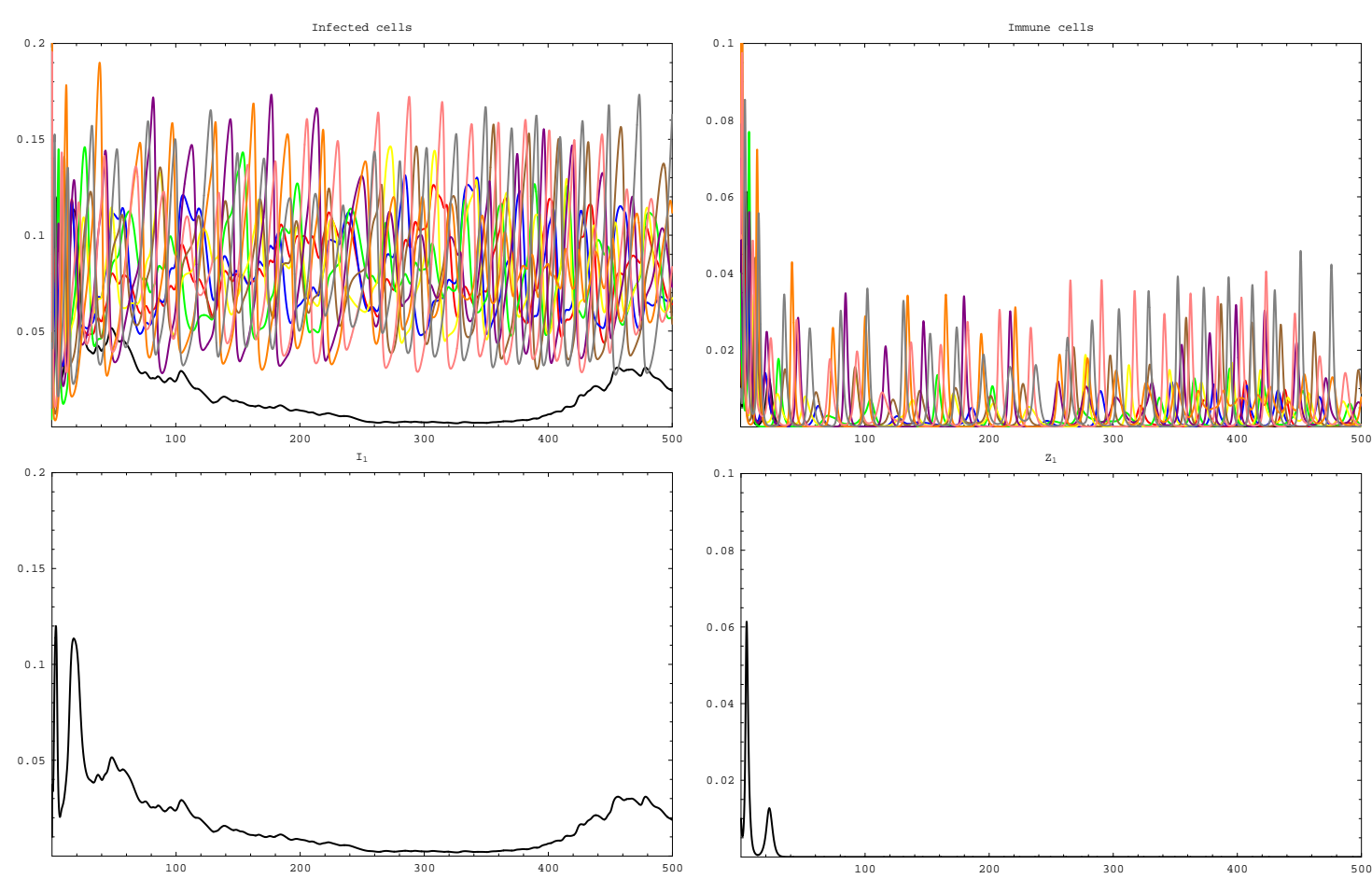

Figure 2: All HIV strains coexist but one of the immune strain $Z_{1}$ becomes inactivated after the mutant HIV strain $I_{10}$ is introduced. In this case, $E_{10}^{+}$can not exist but $\bar{E}_{10}$ exists because $n>n^{*}$ is satisfied . The solution of (2) eventually converges to $\bar{E}_{10}$. This implies that the immune system can not maintain immune diversity and one of HIV strains, $I_{1}$, escapes from its specific immune response $Z_{1}$. These phenomena are caused by the accumulation of viral diversity. Lower panels represent $I_{1}$ (left) and $Z_{1}$ (right). The horizontal axis represents time.

unstable. A computer simulation is shown in Fig.2. Although the dynamics looks like chaotic again, the solution eventually converges to $\bar{E}_{10}$. All HIV strains coexist but the immune strain $Z_{1}$ becomes inactivated. Therefore, the HIV strain $I_{1}$ escapes from its specific immune response when the mutant HIV strain $I_{10}$ invades. That is, the immune system can not maintain immune diversity. The HIV strain which has the minimum basic reproductive number can escape when the number of HIV strains exceeds the immune diversity threshold.

\section{Discussion}

In this study, we consider the effect of viral diversity on the human immune system with the frequency dependent proliferation rate of CTLs and the elimination rate of infected cells by CTLs. In [8], we have studied the complex behavior of (2) for 
$n=1,2$. In this paper, we have especially investigated the properties of (2) for large number of HIV strains and the effect of the accumulation of viral diversity.

The most important idea in this paper is "immune diversity threshold". We can obtain the number of the maximum immune diversity. The previous viral diversity threshold theory proposed by Martin A. Nowak explains the viral explosion by the viral diversity. He concluded that the virus population can no longer be controlled by immune system if the diversity (a Simpson index, which is an inverse measure for viral diversity) exceeds some threshold value. The diversity threshold is caused by an effect of HIV impairment to immune response and characterized by both immune and viral ability. His model has three different parameter regions, which correspond to the observed patterns of HIV and SIV infection: (i) rapid progression to disease and death, (ii) asymptomatic infection without disease, and (iii) development of disease after a long and variable asymptomatic period. However, in our theory, immune diversity threshold gives the capacity of immune response and leads to an escape of the nonmutational existing strain from its specific immune response. We can prove the HIV strain with the minimum basic reproductive number can escape from its specific immune response after the viral diversity exceeds the threshold value. The threshold is only characterized by the parameters $c$ and $\delta$ which express the immune ability.

Our model may be thought to be a special case because there is no difference among the immune ability for each immune cell. Even if the immune ability for each immune cell and virological attributes for each HIV strain are not the same, we can also obtain the immune diversity threshold. For example, we assume that each specific immune cell has a different immune efficiency and decay rate:

$$
Z_{j}^{\prime}=c_{j} Z_{j} \frac{I_{j}}{T+\sum_{l=1}^{n} I_{l}}-\delta_{j} Z_{j}
$$

Then we can have the immune diversity threshold in the similar manner:

$$
1-\sum_{l=1}^{n} \frac{\delta_{l}}{c_{l}}>0 .
$$

If this condition does not hold, then the interior equilibrium can not exist in the nonnegative cone. Therefore the accumulation of viral diversity can lead to the immune inactivation. Note that the difference of virological attributes for HIV does not change the immune diversity threshold but changes existence conditions of equilibria. Thus the different virological attributes do not affect our diversity implications for HIV infection.

Next we consider whether the accumulation of viral diversity leads to AIDS or the end of the asymptomatic phase. Immunity is usually divided into two basic 
types, that is, innate and adaptive. Usually, two basic types of immunity are innate and adaptive. The innate immunity such as macrophage and NK cell activation are the first line of the immune system. Innate immunity can eliminate the pathogen in most cases, but sometimes it fails. Actually HIV can break this defense line. When innate immunity fails, adaptive immunity can be induced. This immune response is characterized by the $\mathrm{T}$ cell and $\mathrm{B}$ cell response. The adaptive immune cell is specific to pathogen because the immune response is tailored to the type of pathogen. However it takes at least 96 hours for sufficient numbers of adaptive immune cells to be produced because of activation of co-stimulatory molecules of dendritic cells, the signal induced upon recognition by the innate immune system and so on [2], [13]. In HIV infection, HIV specific immune cell which can down-regulate the virus is activated in the end of primary phase but the immune cell can not completely eliminate HIV in vivo. Furthermore, there are ample experimental evidences for HIV infection: the HIV population in any one infected host is fairly homogeneous during primary phase but becomes heterogeneous afterwards; the average life-cycle of HIV during the asymptomatic phase of infection is short, about 1-2 days; hence the mutant HIV strain can rapidly induce its specific immune response; HIV escapes from B cell and T cell mediated immune response [11], [12]. Therefore, we assume that HIV can mutate during the course of infection, and the mutant can escape from the existing immune responses and induce its specific immune response. In [8], we showed that viral diversity can lead to the collapse of the immune system because of complex dynamics observed for (2). However, in this paper, we assume that the solution converges to some equilibrium in order to investigate the effect of viral diversity. We consider that the course of HIV infection with mutation corresponds to the following shift of equilibrium to which the solution converges:

$$
E_{0} \Longrightarrow E_{1} \Longrightarrow E_{1}^{+} \Longrightarrow E_{2}^{+} \Longrightarrow E_{3}^{+} \Longrightarrow \cdots
$$

We assume that the specific immune response corresponding to the mutant viral strain is immediately induced when the mutant strain appears. Therefore, the progression in asymptomatic phase can be represented by the shift of $E_{c}$-type euilibria. Remark that $E_{0}=(1,0,0)$ represents the healthy state with the only uninfected $\mathrm{CD}^{+} \mathrm{T}$ cells, $E_{1}=\left(a / \beta_{1}, 1 / a-1 / \beta_{1}, 0\right)$ represents the beginning of primary phase of HIV infection and $E_{1}^{+}$represents the end of primary phase.

In the case of virtual experiment, the disease progression corresponds to the following shift of equilibrium:

$$
E_{0} \Longrightarrow E_{1} \Longrightarrow E_{1}^{+} \Longrightarrow E_{2}^{+} \cdots E_{9}^{+} \Longrightarrow \bar{E}_{10} .
$$


In this case, we can consider that $\bar{E}_{10}$ represents the end of asymptomatic phase or the onset of immunodeficiency phase. This is because the number of $\mathrm{CD}^{+} \mathrm{T}$ cell decreases to the level without any immune activation which corresponds to the low $\mathrm{CD}^{+} \mathrm{T}$ cell level in the beginning of primary phase $E_{1}$. We plot the size of uninfected $\mathrm{CD}^{+} \mathrm{T}$ cells and the size of total infected $\mathrm{CD} 4^{+} \mathrm{T}$ cells at equilibria for (7) by using (3) and (4) with the same parameters as Fig.2 in order to obtain the detailed information of disease progression in terms of our mathematical model. In
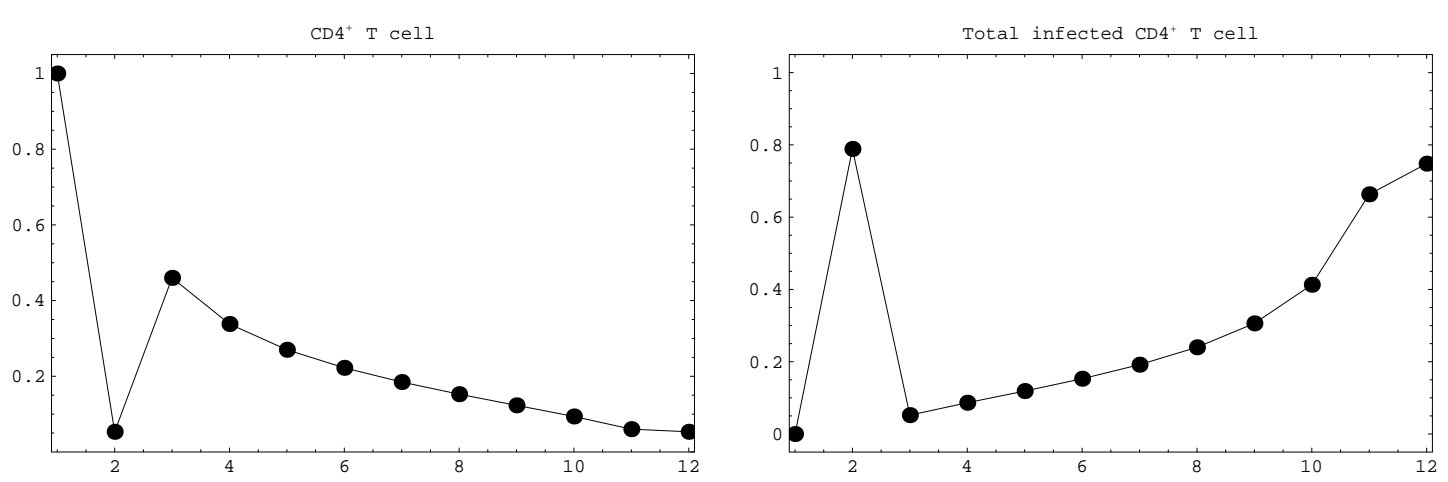

Figure 3: The disease progression (7) of HIV infection: We plot the size of uninfected $\mathrm{CD} 4^{+} \mathrm{T}$ cells and the size of total infected CD4 ${ }^{+} \mathrm{T}$ cells at equilibria for $(7)$. The left figure expresses the shift of uninfected $\mathrm{CD} 4^{+} \mathrm{T}$ cell size. The right figure expresses the shift of total infected $\mathrm{CD} 4^{+} \mathrm{T}$ cell size. These figures represent a typical disease progression of HIV infection. The parameters are the same as Fig.2. The horizontal axis represents the progression time of the HIV infection.

Fig.3, the left figure expresses the shift of uninfected CD4 ${ }^{+} \mathrm{T}$ cell size. From left to right, the first bullet corresponds to the size of uninfected $\mathrm{CD} 4^{+} \mathrm{T}$ cell at $E_{0}$ and the second one corresponds to one at $E_{1}$ and the third one corresponds to one at $E_{1}^{+}$. In the similar manner, the twelfth bullet corresponds to one at $\bar{E}_{10}$. The right figure expresses the shift of total infected $\mathrm{CD} 4^{+} \mathrm{T}$ cell size in the same order. We regard that (7) denotes the disease progression. A typical progression of HIV infection is as follows: The first few weeks after inoculation with virus mark the primary phase of the infection. Patients usually develop high virus loads $\left(E_{1}\right)$. CD4 ${ }^{+} \mathrm{T}$ cells fall transiently and then slightly increase $\left(E_{1} \rightarrow E_{1}^{+}\right)$. At the end of the primary phase, virus load falls again $\left(E_{1}^{+}\right)$; After this phase, patients enter the second phase of HIV disease which is largely asymptomatic $\left(E_{2}^{+} \rightarrow \cdots \rightarrow E_{9}^{+}\right)$. Fig.3 represents a typical disease progression of HIV infection such as the acute phase, the asymptomatic phase and the onset of immunodeficiency phase. In virtual experiment (in Fig.3), the viral diversity is accumulated by an evolutionary event and the accumulation of viral diversity leads to the end of asymptomatic phase because of the inactivation of 
the specific immune response and the number of $\mathrm{CD} 4^{+} \mathrm{T}$ cell. Thus the occurrence of an escape of the nonmutational existing strain from its specific immune response denotes the end of asymptomatic phase or the onset of immunodeficiency phase. Although the average length of the asymptomatic period is about ten years, there is a long and variable asymptomatic phase. In terms of our theory, the variable length of the asymptomatic period is characterized by the ratio of the maximum efficiency of immune inducement to the decay rate of CTLs. For instance, a patient who has large $c$ tends to have high immune diversity and to delay the progression of the disease. On the other hands, a patient who has small $c$ tends to have low immune diversity and to accelerate the disease progression. These phenomena can be observed in [23], [25]. Hence our theoretical study suggests that an effective immunotherapy should be towards increasing the proliferation rate of CTLs $c$ or reducing the death rate of CTLs $\delta$. High-level expression of programmed death 1 (PD-1) on the surface of HIV-specific T cells is associated with the downregulation of proliferation and cytokine production of $\mathrm{T}$ cells, which consequently induces CD8 T-cell dysfunction and disease progression. Moreover, the level of PD-1 surface expression is the primary determinant of apoptosis sensitivity of virus-specific CD8 ${ }^{+}$ T cells. Manipulation of PD-1 leads to changes in the ability of the cells to survive and expand [1], [24], [27]. Thus, according to our theory, blocking the PD-1-PD-L1 pathway is suggested to be a very effective therapy because along with this therapy not only the proliferation rate of CTLs would increase but also the death rate of CTLs would decrease.

The final phase of the disease is characterized by the development of AIDS. Usually a healthy human adult has about 1000 CD4 cells per $\mu$ l of blood, but in an HIV infected patient the abundance of CD4 cells can decline to very low numbers. A CD4 cell count of less than 200 per $\mu \mathrm{l}$ is currently used as definition for AIDS regardless of the presence of opportunistic infections [13], [19], [23]. We consider an additional mutant HIV strain $\left(I_{11}\right)$ satisfying with $\beta_{10}<\beta_{11}$ (this assumption is not essential for our result) by the evolutionary event after the disease progression of (7). What happens if the additional mutant occurs? In this case, the shift of immune response can be observed. The shift of immune response means that the specific immune response to a mutant HIV strain is activated by the introduction of the mutant HIV strain but wild-type specific immune response becomes inactivated, although the corresponding wild-type HIV strain remains. That is, the structure of the HIV strains and their specific immune cells changes. This is caused by competition among HIV strains to the uninfected CD4 ${ }^{+} \mathrm{T}$ cells. Since the number of HIV strains exceeds the immune diversity threshold, new controlled equilibrium $E_{11}^{+}$can 
not bifurcate. The solution converges the following $E_{u}$-type equilibrium:

$$
\hat{E}_{11}=\left(\hat{T} ; 0, \hat{I}_{2}, \ldots, \hat{I}_{11} ; 0,0, \hat{Z}_{3}, \ldots, \hat{Z}_{11}\right) .
$$

Note that the number of active immune strains does not change, although the strain structure changes from $\bar{E}_{10}$. However the abundance of uninfected CD4 ${ }^{+} \mathrm{T}$ cells decreases and infected $\mathrm{CD} 4^{+} \mathrm{T}$ cells increase. Further the additional mutation of HIV strain leads to the $E_{u}$-type equilibrium, to the shift of immune response and to some deterioration of symptom in the similar manner. In fact, the number of CD4 ${ }^{+}$ $\mathrm{T}$ cell can be represented by $a / \beta_{k-\left[n^{*}\right]}$ if the $E_{u}$-type equilibrium exists when the HIV strain $k>\left[n^{*}\right]$ appears. In general, we can conclude that $a / \beta_{k-\left[n^{*}\right]}$ monotonically decreases as $k$ increases because of $\beta_{1}<\beta_{2}<\ldots<\beta_{n}$. This implies that the CD4 ${ }^{+}$ T cell count essentially drops to 0 during the course of infection. That is, we can conclude that the viral diversity eventually leads to AIDS. Thus the evolutionary events can tend to progress HIV infection even if the number of active immune strains does not increase. This new idea can explain the limit of immune system in human against HIV.

Note that our theory does not represent that the strain with the minimum basic reproductive number will cause immune collapse and lead to disease progression. Our finding demonstrates that the emergence of invasive HIV strain which has a larger basic reproductive number leads to the disease progression. Actually transmission and early asymptomatic phase of HIV are related to the R5 strain (that later on mutates to the more virulent X4 strain) and rapid disease progression and $\mathrm{CD}^{+}{ }^{+} \mathrm{T}$ cell depletion is associated with the emergence of the X4 strain [13]. Further our theory does not also represent that high immune ability (high $c$ and low $\delta$ ) which potentially leads to high immune diversity always guarantees a slow progression of the disease. We can find in our model the effect of tareget cell $\left(\mathrm{CD} 4^{+} \mathrm{T}\right.$ cell) limitation which is the same as the results obtained in [25]. In this paper, we neglect this effect by assuming the existence of $E_{c}$-type equilibrium related with $\left[n^{*}\right]$ in order to investigate the relation between viral diversity and immune ability. This implies that virological attributes also contribute the limitation of immune diversity and the disease progression.

In our mathematical model (1), the cells infected with one HIV strain are only eliminated by one type of CTL. In reality different HIV strains do not have completely different epitopes and many types of CTL will recognize the majority of infected cells even though they are infected with different strains. Therefore, we should consider the cross reactivity of CTL. It is expected that cross reactivity may tend to reduce the likelihood of chaotic behavior observed in [8], and change the 
diversity threshold and a distribution of equilibria. Moreover we should consider a direct impairment effect of HIV to immune response. These are our future works. Although our model does not include these effects and immune diversity threshold needs to be treated with caution, our theory captures the essence of HIV disease progression and suggests the mechanism of immunodeficiency.

\section{Acknowledgements}

The authors express thanks to Prof. Yoshiharu Miura and Prof. Yoshio Koyanagi for valuable comments. And also we would like to thank anonymous referees for very helpful suggestions and comments which improved the quality of this paper and study.

\section{References}

[1] D.L. Barber, E.J. Wherry, D. Masopust, B. Zhu, J.P. Allison, A.H. Sharpe, G.J. Freeman, R. Ahmed (2006), Restoring function in exhausted CD8 T cells during chronic viral infection. Nature, 439, 682-687.

[2] E. Beretta, M. Carletti, D. E. Kirschner and S. Marino (2006), Stability analysis of a mathematical model of the immune response with delays, Mathematics for Life Science and Medicine, (Eds. Y. Takeuchi, Y. Iwasa, K. Sato), Springer: New York, 177-206.

[3] D. M. Davis (2006), Intrigue at the immune synapse, Sci. Amer., February.

[4] M. L. Dustin and D. R. Colman (2002), Neural and immunological synaptic relations, Scince, 298, 785-789.

[5] A. Fomsgaard (1999), HIV-1 DNA vaccines, Immunology letters, 65, 127-131.

[6] S. D. W. Forst and C. A. Michie (1996), Lymphocyte dynamics, apoptosis and HIV infection, Trends in microbiology, 4, 77-82.

[7] P. J. R. Goulder, R. E. Phillips, R. A. Colbert, S. McAdam, G. Ogg, M. A. Nowak, P. Giangrande, G. Luzzi, B. Morgan, A. Edwards, A.J. McMichael and S. Rowland-Jones (1997), Late escape from an immunodominant cytotoxic Tlymphocyte response associated with progression to AIDS, Nature medicine, 3 , 212-217. 
[8] S. Iwami, S. Nakaoka, Y. Takeuchi (2006), Frequency dependence and viral diversity imply chaos in HIV model, Physica D, 223, 222-228

[9] S. Iwami, S. Nakaoka, Y. Takeuchi (preprint), Mathematical analysis of HIV model with frequency dependence and viral diversity.

[10] Y. Iwasa, F. Michor and M. A. Nowak (2004), Some basic properties of immune selection, J. theor Biol., 229, 179-188.

[11] Y. Iwasa, F. Michor and M. A. Nowak (2005), Virus evolution within patients increases pathogenicity, J. theor Biol., 232, 17-26.

[12] Y. Iwasa, F. Michor and M. A. Nowak (2006), Directional evolution of virus within a host under immune selection, Mathematics for Life Science and Medicine, (Eds. Y. Takeuchi, Y. Iwasa, K. Sato), Springer: New York, 155176.

[13] C. Janewa, P. Travers, M. Walport, M. J. Shlomchik (2004), Immunobiology: The Immune System in Health and Disease, Garland Pub.

[14] G. L. Pira, L. Bottone, D. Fenoglio, P. Terranova, E. Pontali, F. Ivaldi, F. D. Galdo, L. Mortara, A. Loregian, G. Palu, A. Kunkl, R. Accolla, R. D. Palma, F. Manca (2001), Analysis of the antigen specific T cell repertoires in HIV infection, Immunology Letter, 79, 85-91.

[15] A. McKnight and P. R. Clapham (1995), Immune escape and tropism of HIV, Trends in microbiology, 3, 356-361.

[16] A. J. McMichael (1998), T cell response and viral escape, Cell, 93, 673-677.

[17] A. J. McMichael and S. L. Rowland-Jones (2001), Cellular immune responses to HIV, Nature, 410, 980-987.

[18] M. A. Nowak (1992), Variability in HIV infections, J. theor Biol., 155, 1-20.

[19] M. A. Nowak (2006), Evolutionary dynamics, Harvard University Press.

[20] M. A. Nowak, R. M. Anderson, A. R. McLean, T. Wolfs, J. Goudsmit and R. M. May (1991), Antigenic diversity thresholds and the development of AIDS, Science, 254, 963-369.

[21] M. A. Nowak and R. M. May (1991), Mathematical biology of HIV infections: antigenic variation and diversity threshold, Math Biosci, 106, 1-21. 
[22] M. A. Nowak and R. M. May (1993), AIDS pathogenesis: mathematical models of HIV and SIV infections, AIDS, 7, S3-S18.

[23] M. A. Nowak and R. M. May (2000), Virus Dynamics, Oxford University Press.

[24] C. Petrovas, J.P. Casazza, J.M. Brenchley, D.A. Price, E. Gostick, W.C. Adams, M.L. Precopio, T. Schacker, M. Roederer, D.C. Douek, R.A. Koup (2006), PD1 is a regulator of virus-specific CD8+ T cell survival in HIV infection, J. Exp. Med. 203, 2281-92.

[25] R. R. Regoes, D. Wodarz and M. A. Nowak (1998), Virus dynamics: the effect of target cell limitation and immune responses on virus evolution, $J$ theor Biol, 191, 451-462.

[26] X. Xu, G. R. Screaton and A. J. McMichael (2001), Virus Infections: Escape, Resistance, and Counterattack, Immunity, 15, 867-870.

[27] L. Trautmann, L. Janbazian, N. Chomont, E.A. Said, S. Gimmig, B. Bessette, M.R. Boulassel, E. Delwart, H. Sepulveda, E.S. Balderas, J.P. Routy, E.K. Haddad, R.P. Sekaly (2006), Upregulation of PD-1 expression on HIV-specific CD8+ T cells leads to reversible immune dysfunction, Nature Medicine 12, 1198-202.

[28] D. Wodarz and A. R. Thomsen (2005), Does programmed CTL proliferation optimaize virus contorol, Trends in Immunology, 26, 305-310. 